\title{
Reconstrução arterial com tubo de pericárdio bovino corrugado
}

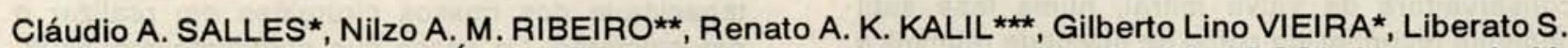
S. SOUZA*, Paulo M. BORÉM*, Miguel E. C. ANDRADE*, Rogerio D. FALEIROS*, Marcos A. M. ANDRADE Jr.*

\section{RBCCV 44205-166}

SALLES, C. A.; RIBEIRO, N. A. M.; KALIL, R. A. K.; VIEIRA, G. L.; SOUZA, L. S. S.; BORÉM, P. M.; ANDRADE, M. E. C.; FALEIROS, R. D.; ANDRADE Jr., M. A. M. - Reconstrução arterial com tubo de pericárdio bovino corrugado. Rev. Bras. Cir. Cardiovasc., 7(2):88-95, 1992.

RESUMO: No período de agosto de 1989 a fevereiro de 1992, 32 pacientes foram submetidos a reconstruçöes vasculares utilizando-se condutos de pericárdio bovino corrugado preservado em glutaraldeído. A incorporação do príncipio crimping utilizado nas próteses vasculares sintéticas proporcionou tubos que mantêm sua forma cilíndrica, mesmo quando submetidos a curvaturas. Vinte e nove pacientes (Grupo l) eram portadores de doenças da aorta torácica e/ou abdominal, incluindo aneurismas, dissecçōes agudas, coarctaçāo da aorta e lesāo oclusiva aorto-ilíaca. A reconstruçāo da aorta torácica foi realizada em 25 pacientes ( incluindo a substituiçāo da valva aórtica em 10), da aorta abdominal em 2 e aorto-ilíaca em 2 . Três pacientes (Grupo II), portadores de cardiopatias congênitas complexas, foram submetidos a reconstrução da via de saída do ventrículo direito em 2 e a operaçāo de Fontan em 1. A mortalidade hospitalar no Grupo I fol $24 \%$ (7 pacientes), causada por baixo débito cardíaco em 4, recidiva precoce da dissecção em dois e infecçāo respiratória em 1. Seis destes óbitos ocorreram em pacientes operados na fase aguda de dissecção aórtica. Não houve nenhum óbito no Grupo II. Houve um óbito tardio no Grupo I devido a complicaçōes metabólicas relacionadas a diabetes e insuficiência renal crônica. Esta experiência clínica inicial registrou um seguimento médico de 16 meses por paciente, com um máximo de 32 meses e não se verificou nenhuma complicaçāo tardia relacionada ao conduto de pericárdio bovino corrugado.

DESCRITORES: prótese arterial; prótese vascular; aneurismas de aorta, cirurgia.

\section{INTRODUÇĀO}

A interposiçāo de um enxerto para restabelecer a continuidade da aorta foi relatada no final da década de 1940 e início da década de 1950, utilizando-se enxertos arteriais humanos preservados $22,25,38$. A prótese vascular sintética porosa foi introduzida em 1952, utilizando-se o Vinyon $\mathrm{N}^{49} \mathrm{e}$ vários outros materiais biocompatíveis foram empregados em estudos experimentais e investigaçōes clínicas. Dacron foi considerado o material de escolha para a fabricação de próteses vasculares e tem sido amplamente utilizado nas últimas décadas ${ }^{31,} 32$
O pericárdio autógeno ${ }^{40}$ e enxertos aórticos alógenos ${ }^{41}$ sāo empregados desde 1965 , para a reconstrução da via de saída do ventrículo direito, seguidos pela utilização de um tubo de Dacron contendo uma bioprótese porcina ${ }^{2}$. No entanto, problemas relacionados com disponibilidade, preservaçāo e alguns aspectos técnicos com referência aos enxertos aórticos humanos e a obstruçāo observada nos tubos de Dacron têm limitado o seu uso 2, 21, 26 .

A aorta ascendente, o arco aórtico, a aorta descendente torácica e abdominal podem ser recons-

Trabalho realizado no Hospital Felício Rocho, Belo Horizonte, MG, Hospital Santa Isabel, Salvador, BA e Instituto de Cardiologia, Porto Alegre, RS, Brasil.

Apresentado ao $19^{\circ}$ Congresso Nacional de Cirurgia Cardíaca. Sáo Paulo, SP, 7 a 9 de maio, 1992.

Laureado com o Prêmio Nacional de Cirurgia Cardíaca - 1992.

* Do Hospital Felício Rocho de Belo Horizonte.

* Do Hospital Santa Isabel de Salvador.

* Do Instituto de Cardiologia de Porto Alegre.

Endereço para separatas: Cláudio A. Salles. Av. Celso Porfirio Machado, 620. 30330 Belo Horizonte, MG, Brasil. 

R. D.; ANDRADE Jr., M. A. M. - Reconstruçäo arterial com tubo de pericárdio bovino corrugado. Rev. Bras. Cir. Cardiovasc., $7(2): 88-95,1992$.

truídos através de várias técnicas, utilizando-se próteses tubulares de Dacron $1,5,7,8,14,18,19,34,39$. A correçẳo de uma dissecção aórtica é freqüentemente complicada por hemorragias severas, resultantes de sangramento na linha de sutura entre o Dacron e a parede friável da aorta $1,14,29,30,34$.

O pericárdio bovino preservado em glutaraldeído tem sido utilizado no Brasil desde 1979, para alargamento da aorta ascendente ${ }^{10}$, seguido do uso de condutos de pericárdio contendo uma bioprótese para substituiçāo total da aorta ascendente ${ }^{4}$. O pericárdio é macio e flexível, o que facilita consideravelmente a feitura da anastomose com uma aorta fina e friável, resultando uma linha de sutura mais hemostática e reduzindo os problemas de sangramento quando comparado com os tubos de Dacron ${ }^{4}$

Em 1987, foi desenvolvido um conduto de pericárdio bovino incorporando o príncipio crimping utilizado nas próteses vasculares de Dacron ${ }^{5}$. Detalhes técnicos do preparo do pericárdio e da confecção dos tubos, assim como a avaliação da integridade bioquímica, estrutural e funcional do tecido biológico foram descritos previamente ${ }^{43}$. O processo crimping proporciona um tubo cilíndrico corrugado que mantém a sua forma tubular, mesmo quando fletido, facilitando a sua manipulação durante a cirurgia e a realização das anastomoses, além de proporcionar uma elasticidade longitudinal (Figura 1).

A experiência clínica inicial demonstrou uma reduçăo significativa do sangramento intra e pósoperatório e ausência de complicaçōes decorrentes do uso desse conduto de pericárdio bovino corrugado $42 \cdot 44$

\section{CASUÍSTICA E MÉTODOS}

No período de agosto de 1989 a fevereiro de 1992, 32 pacientes foram submetidos a reconstruçōes vasculares, utilizando-se condutos de pericárdio bovino corrugado preservado em glutaraldeído, manufaturados industrialmente e disponíveis no mercado em diferentes tamanhos e modelos, avalvulados ou compostos, contendo uma bioprótese valvular (Figura 2).

Vinte e nove pacientes (Grupo I) eram portadores de lesōes aórticas, incluindo aneurismas, dissecçōes agudas, coarctação ou lesōes oclusivas (Tabela 1). Dois deles haviam sido submetidos a cirurgias cardiacas prévias. Treze destes pacientes foram admitidos em caráter de urgência devido a dissecção aórtica aguda tipo I em 6, dissecção aórtica aguda tipo III em 6 e aneurisma da aorta abdominal em expansāo em 1 . Insuficiência coronária aguda estava presente em 2 destes pacientes; em um dos casos de dissecção aórtica aguda tipo III havia uma rotura associada. Os outros 16 pacientes do Grupo I foram admitidos para cirurgias eletivas. Os diagnósticos pré-operatórios foram confirmados pela aortografia em todos os pacientes. A idade variou entre 23 e 73 anos, com média de 55 anos. Havia 21 pacientes do sexo masculino e 8 do sexo feminino.

Três pacientes do Grupo II foram encaminhados para correçāo de cardiopatias congênitas complexas, incluindo uma criança de 3 anos, sexo masculino, com ventrículo único, que havia sido submetida previamente a correçāo de coarctaçāo da aorta e cerclagem do tronco pulmonar, uma criança de 4 anos, sexo feminino, com atresia pulmonar, comunicaçāo interventricular e persistência do canal ar-

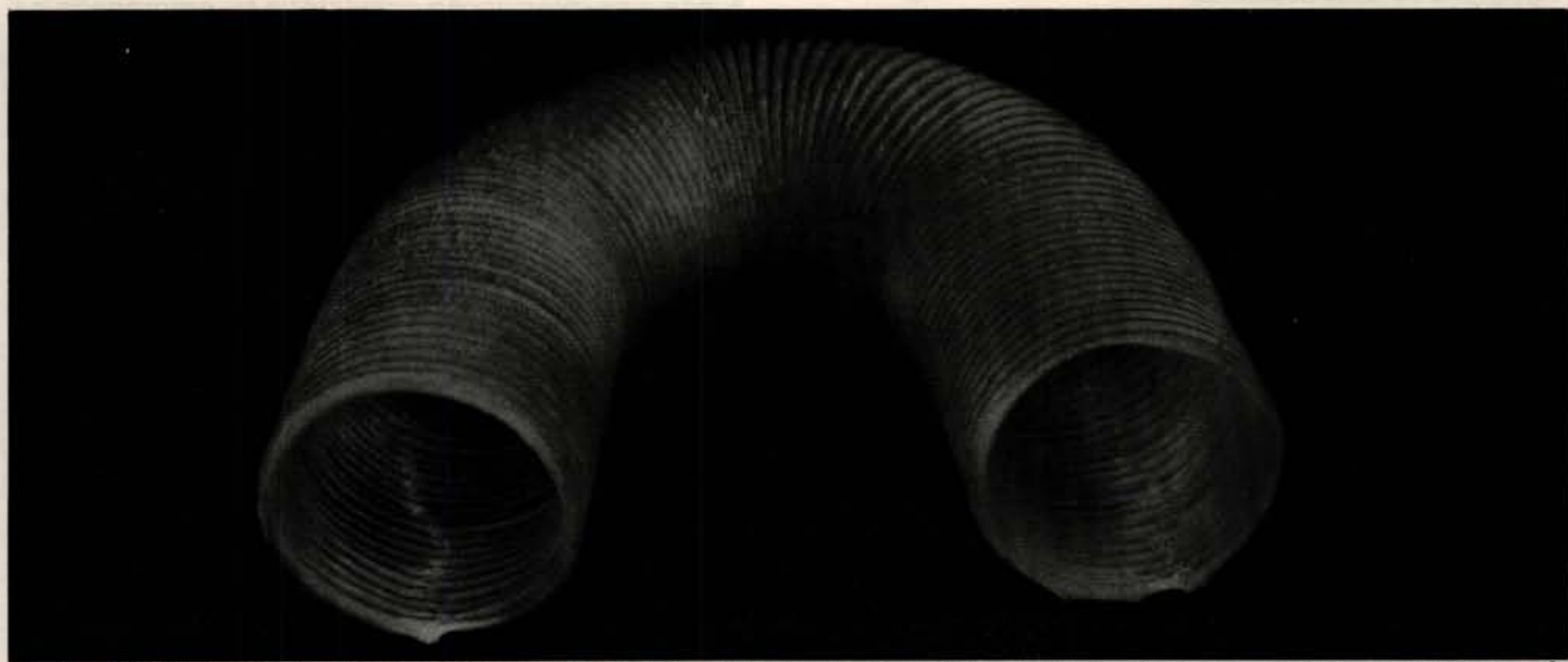

Fig. 1 - Conduto de pericárdio bovino corrugado preservado em glutaraldeído. 0 princípio crimping permite que o conduto seja curvado a $180^{\circ}$, mantendo a sua forma tubular cilíndrica e evita o kinking. 
SALLES, C. A.; RIBEIRO, N. A. M.; KALIL, R. A. K.; VIEIRA, G. L.; SOUZA, L. S. S.; BORÉM, P. M.; ANDRADE, M. E. C.; FALEIROS, R. D.; ANDRADE Jr., M. A. M. - Reconstruçāo arterial com tubo de pericárdio bovino corrugado. Rev. Bras. Cir. Cardiovasc., $7(2): 88-95,1992$.

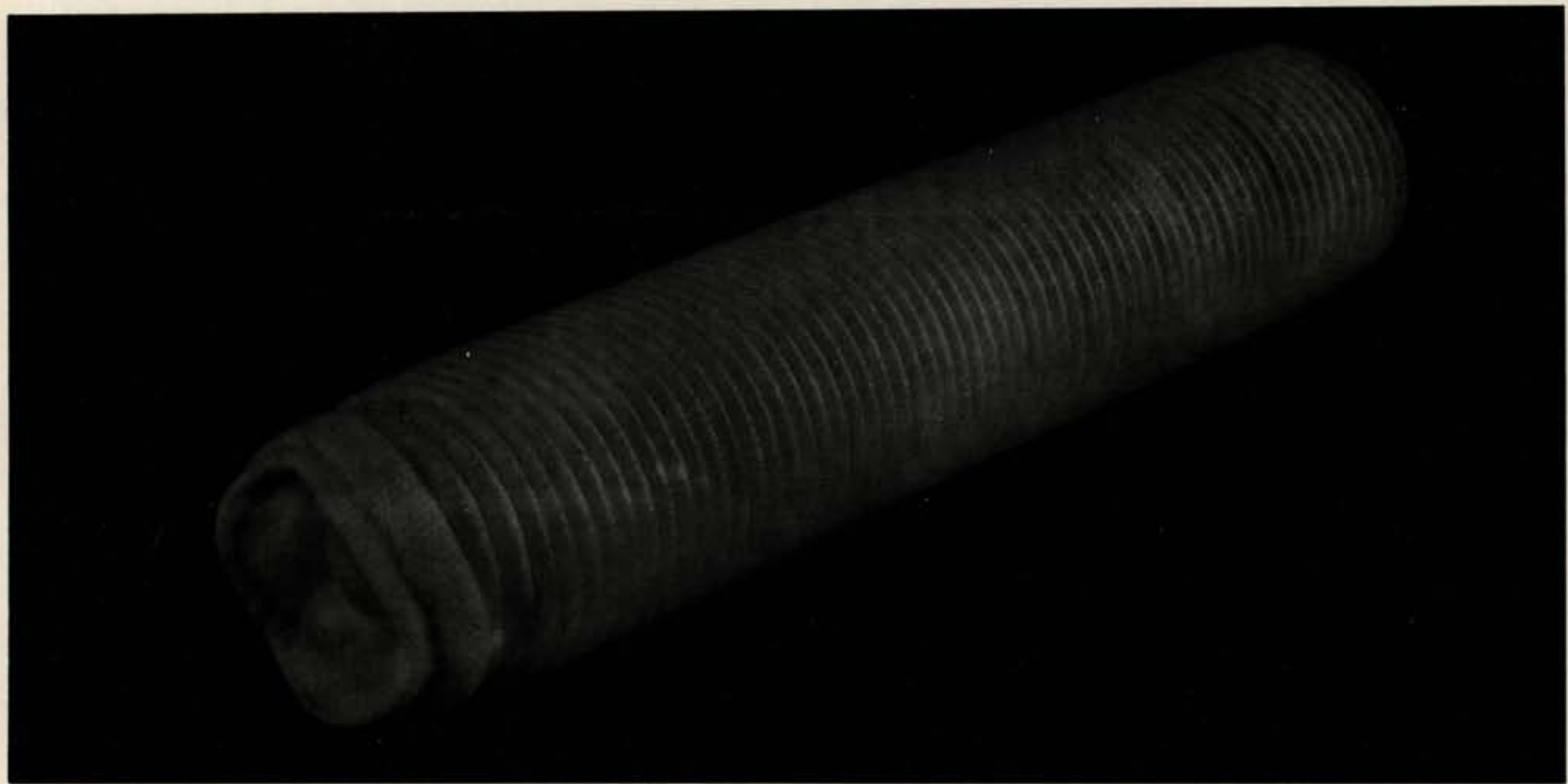

Fig. 2 - Conduto de pericárdio bovino corrugado contendo uma bioprótese aórtica de porco, utilizado para reconstrução total da aorta ascendente.

TABELA 1

DIAGNÓSTICO PRÉ-OPERATÓRIO DOS PACIENTES DO GRUPO I

\begin{tabular}{lc}
\hline \multicolumn{1}{c}{ DIAGNÓSTICO } & $\begin{array}{c}\text { N DE } \\
\text { PACIENTES }\end{array}$ \\
\hline Ectasia ânulo-aórtica & 8 \\
Dissecção aórtica tipo I & 6 \\
Dissecção aórtica tipo III & 6 \\
Aneurisma da aorta ascendente & 2 \\
Aneurisma da aorta torácica descendente & 2 \\
Coarctaçāo da aorta torácica & 1 \\
Aneurisma da aorta abdominal & 3 \\
Obstrução aorto-ilíaca & 1 \\
\hline TOTAL & 29 \\
\hline
\end{tabular}

terial, e 1 paciente de 16 anos, sexo masculino, com uma forma extrema de tetralogia de Fallot.

\section{Procedimentos Cirúrgicos}

A Tabela 2 mostra as operaçōes realizadas nos pacientes do Grupo I. A ectasia ânulo-aórtica, aneurisma da aorta ascendente e dissecçāo aórtica tipo I foram abordadas através da esternotomia mediana, utilizando-se circulaçāo extracorpórea, hipotermia sistêmica e cardioplegia. Tubos de pericárdio bovino corrugado valvulado foram utilizados para substituiçăo total da aorta ascendente, incluindo a valva aórtica e reimplante das artérias coronárias, empregando-se a técnica originalmente descrita por BENTALL \& DE BONO ${ }^{5}$. Condutos avalvulados foram utilizados para substituiçāo da aorta ascendente quando a valva aórtica foi preservada. Nas dissecçōes aórticas, o falso lume foi obliterado proximalmente' e distalmente com o uso da cola biológica (gelatina-resorcina polimerizada pelo formaldeído) e por pontos separados em $U$ reforçados com tiras de pericárdio bovino colocadas por dentro e por fora da aorta. Foi, também, feita cerclagem da anastomose distal com a utilizaçăo de uma fita de pericárdio bovino. A hipotermia profunda com interrupção circulatória foi empregada na correção de quatro casos de dissecçāo aórtica tipo III, utilizando-se a técnica da endoprótese (tromba de elefante) descrita por BORST \& SCHAPS ?, e

TABELA 2

OPERAÇÕES REALIZADAS NOS PACIENTES DO GRUPO I

\begin{tabular}{lc}
\hline PROCEDIMENTO CIRÚRGICO & $\begin{array}{c}N^{0} \text { DE } \\
\text { PACIENTES }\end{array}$ \\
\hline Operação de Bentall e De Bono & 10 \\
Substituição da aorta ascendente & 6 \\
$\begin{array}{l}\text { Substituição da aorta torácica descendente } \\
\text { Endoprótese na aorta torácica descendente } \\
\quad \text { (tromba de elefante) }\end{array}$ & 5 \\
Substituição da aorta abdominal infra-renal & 4 \\
Substituição do seguimento aorto-ilíaco & 2 \\
\hline TOTAL & 2 \\
\hline
\end{tabular}


SALLES, C. A.; RIBEIRO, N. A. M.; KALIL, R. A. K.; VIEIRA, G. L.; SOUZA, L. S. S.; BORÉM, P. M.; ANDRADE, M. E. C.; FALEIROS, R. D.; ANDRADE Jr., M. A. M. - Reconstruçäo arterial com tubo de pericárdio bovino corrugado. Rev. Bras. Cir. Cardiovasc., 7(2):88-95, 1992.

BOAST et alii ${ }^{8}$ e na correção de 1 caso de dissecção aórtica tipo I. A revascularizaçāo miocárdica através de pontes de veia safena foi associada em 2 destes pacientes.

A toracotomia póstero-lateral esquerda através do $4^{2}$ espaço interconstal foi utilizada em 2 pacientes com aneurisma da aorta torácica descendente, em 2 com dissecção aórtica tipo III e na coarctação da aorta. A laparatomia mediana foi usada para abordagem das lesōes da aorta abdominal e do segmento aorto-ilíaco. Os procedimentos sobre a aorta torácica descendente e aorta abdominal foram realizados com simples pinçamentos da aorta empregando-se heparinizaçāo sistêmica.

As anastomoses foram realizadas com fio de Polipropileno 4-0 e não ocorreu nenhum caso de sangramento intra-operatório significativo. As linhas de sutura foram sempre cuidadosamente inspecionadas e utilizados pontos adicionais quando necessário. Subseqüentemente, as paredes do aneurisma foram usadas para recobrir o tubo de pericárdio corrugado e suturadas ao seu redor.

O paciente com ventrículo único foi submetido a inserção de um septo intra-atrial, para excluir a valva tricúspide do átrio direito, e ao implante de um tubo de pericárdio bovino corrugado contendo uma bioprótese de porco entre o átrio direito e o tronco pulmonar. Condutos de pericárdio bovino corrugado foram utilizados para restabelecer a continuidade do ventrículo direito com o tronco pulmonar nos outros 2 pacientes do Grupo II, associado a correção das lesōes intracardíacas e da divisão e sutura do canal arterial persistente, na paciente com atresia pulmonar.

\section{RESULTADOS}

\section{Resultados Imediatos}

A mortalidade hospitalar foi de $24 \%$ ( 7 pacientes) no Grupo I, sendo que 6 destes óbitos ocorreram em pacientes operados de urgência na fase aguda de dissecção aórtica. As causas foram baixo débito cardíaco em 4 , recidiva da dissecção na aorta remanescente em 2 e infecção respiratória em 1.

Três pacientes apresentaram complicaçōes pós-operatórias nāo fatais; 2 desenvolveram atelectasia pulmonar complicada por infecção respiratória, 1 paciente com aneurisma da aorta abdominal em expansāo apresentou insuficiência renal aguda e pancreatite imediatamente após a operação.
Nāo ocorreu óbito nem complicaçōes pósoperatórias significativas entre os pacientes do Grupo II.

\section{Resultados Tardios}

Ocorreu um óbito tardio, três meses após a cirurgia de aneurismectomia da aorta abdominal, em conseqüência de complicaçōes metabólicas relacionadas a insuficiência renal crônica, diabetes e pancreatite.

O seguimento médio entre os 25 sobreviventes da operação foi de 16 meses oor paciente, com um seguimento máximo de 32 meses.

Um paciente submetido a correção de dissecção aórtica tipo I permanece com oclusão parcial da artéria ilíaca direita e claudicação intermitente.

Os 3 pacientes do Grupo II beneficiaram-se, consideravelmente, da correção de suas lesōes cardíacas congênitas com uma significativa melhora do quadro clínico e hemodinâmico.

Não ocorreu nenhuma complicação tardia que pudesse ser relacionada ao tipo de conduto utilizado.

\section{COMENTÁRIOS}

O uso de enxerto para restabelecer a continuidade aórtica foi relatado, inicialmente, por GROSS et alii ${ }^{25}$, em 1949, para correção da coarctação da aorta, utilizando enxertos arteriais humanos congelados a seco. Em 1951, OUDOT ${ }^{38}$ substituiu a bifurcação aórtica e DUBOST et alii ${ }^{22}$ reconstruíram a aorta abdominal distal após a ressecção de um aneurisma; ambos utilizaram enxertos arteriais humanos preservados. Após resultados clínicos iniciais animadores, constatou-se que esses enxertos congelados não viáveis evoluíram para dilatação aneurismática e/ou trombose no intervalo de um a três anos ${ }^{32}$.

Uma prótese vascular sintética porosa (Vinyon N) foi desenvolvida em laboratório, por VOORHEES Jr. et alii ${ }^{49}$, em 1952, e, após estudos experimentais em cães, foi introduzida para uso clínico ${ }^{6}$. Vários outros materiais foram utilizados experimentalmente e clinicamente, durante a década de 1950 , tais como Orlon, Nylon, Ivalon, Teflon e Dacron. A introdução do princípio crimping permitiu aos condutos manter a sua forma cilíndrica, mesmo quando curvados, além de proporcionar uma elasticidade longitudinal. Dacron foi considerado o material de escolha para a confecção de próteses vasculares sintéticas, que foram consideradas muito satisfató- 
SALLES, C. A.; RIBEIRO, N. A. M.; KALIL, R. A. K.; VIEIRA, G. L.; SOUZA, L. S. S.; BORÉM, P. M.; ANDRADE, M. E. C.; FALEIROS, R. D.; ANDRADE Jr., M. A. M. - Reconstruçäo arterial com tubo de pericárdio bovino corrugado. Rev. Bras. Cir. Cardiovasc., $7(2): 88-95,1992$.

rias para a substituiçāo da aorta e das artérias ilíacas. Tornaram-se disponíveis comercialmente e foram confeccionadas sem sutura nas configuraçōes woven, knitted e velour ${ }^{31,32}$. Durante as décadas seguintes, os tubos de Dacron foram amplamente utilizados para corrigir uma grande variedade de lesōes aórticas, incluindo próteses vasculares, compostas contendo uma prótese valvular aórtica para substituição total da aorta ascendente, assim como outros modelos de próteses vasculares $1,5,7$. 8, 14, 18, 19, 29, 34, 39

Em 1965, RASTELLI et alii ${ }^{40}$ relataram o uso de um tubo de pericárdio autógeno avalvular para restabelecer a continuidade entre o ventrículo direito e o tronco pulmonar na correção da atresia pulmonar. Um procedimento similar foi relatado por ROSS e SOMMERVILLE ${ }^{41}$, em 1966, usando um enxerto aórtico alógeno, incluindo a aorta e a valva aórtica. Após esse relato, aloenxertos aórticos congelados e irradiados foram utilizados para correçăo da atresia pulmonar, tronco arterioso, transposiçāo das grandes artérias e outras cardiopatias complexas ${ }^{33}$. Entretando, a incidência de calcificaçāo tardia e obstrução foi alta ${ }^{35}$.

Bons resultados tardios e uma baixa incidência de calcificação têm sido relatados com aloenxertos aórticos valvulados frescos esterelizados em antibióticos, para reconstrução da via de saída do ventrículo direito ${ }^{23,27,28,45,48}$. Problemas relacionados a procura, disponibilidade, preservaçāo e detaIhes técnicos durante a operaçāo têm limitado o seu uso 21,28 .

Em 1973, tubos de Dacron woven contendo uma bioprótese aórtica de porco foram introduzidos para restabelecer a continuidade entre o ventrículo direito e o tronco pulmonar ${ }^{\circ}$. Foram selecionados em diferentes tamanhos e modelos e amplamente usados para corrigir uma grande variedade de lesōes cardíacas ${ }^{11,12,16,24,37}$. Entretanto, o seguimento tardio demonstrou estenose e obstrução dos tubos, particularmente decorrente de fibrose e trombose? Próteses vasculares de Dacron velour impregnadas de colágeno foram introduzidas, para evitar as complicaçōes relatadas com Dacron woven ${ }^{47}$. Apesar disso, obstrução do conduto foi relatada, devido a retraçāo cicatricial e plicatura do tubo por tecido fibroso substituindo o colágeno absorvível ${ }^{3}$.

Um conduto de pericárdio autógeno valvulado confeccionado durante a operação foi descrito por SCHLICHTER e KREUTZER ${ }^{46}$, em 1985, com bons resultados iniciais em 6 pacientes.

O pericárdio bovino tem sido largamente usado para correçāo de lesōes cardíacas congênitas ${ }^{20}$. Embora algumas complicaçōes tenham sido observadas com seu uso como septo atrial ${ }^{13},{ }^{17}$, a retraçāo, a fibrose e a calcificaçāo nāo têm sido mencionadas quando é usado na via de saída do ventrículo direito.

O pericárdio bovino preservado em glutaraldeído tem sido empregado em nosso meio por BRAILE et alii ${ }^{10}$ desde 1979, para alargamento da aorta ascendente. Condutos de pericárdio bovino valvulados foram introduzidos para substituição total da aorta ascendente, incluindo a valva aórtica ${ }^{4} e$ para reconstrução da via de saída do ventrículo direito ${ }^{36}$, sem relatos de complicaçőes até o presente. A flexibilidade e a maciez do tecido biológico facilitam a feitura de uma anastomose hemostática com uma aorta doente. As complicaçōes hemorrágicas diminuíram quando comparadas aos condutos de Dacron ${ }^{4}$, que, muitas vezes, exigem procedimentos cirúrgicos adicionais para controle da hemorragia ${ }^{14,20}$. A parede da aorta na presença de um aneurisma e, especialmente, de uma dissecçāo é muito fina e friável, podendo estar calcificada. A anastomose entre um tubo de Dacron woven espesso e duro e uma aorta friável pode ser difícil, resultando em sangramento na linha de sutura, que algumas vezes é incontrolável e pode ser fatal 1.5, 14. 20, 30, 34. Isto levou ao desenvolvimento de uma prótese vascular intraluminal para casos selecionados de dissecçāo aórtica aguda '.

$\mathrm{Na}$ descriçāo da técnica original de substituiçăo total da aorta ascendente e da valva aórtica, BENTALL e DE BONO ${ }^{5}$ suturaram a aorta aneurismática ao redor do tubo de Dacron para controlar a hemorragia nas linhas de sutura e sangramento intersticial através da malha do conduto. CABROL et alii ${ }^{14}$ criaram uma fístula entre o espaço paraprotético e o átrio direito, após suturar a parede aneurismática da aorta ao redor do tubo de Dacron. Embora esses procedimentos possam ser necessários algumas vezes para promover hemostasia, na presença de um sangramento incontrolável eles podem mascarar pontos importantes de sangramento, resultando complicaçōes tardias, incluindo pseudo-aneurismas, como recentemente mencionado por KOUCHOUKOS ${ }^{29}$. Outros procedimentos empregados para minimizar problemas de sangramento incluem o uso de tubo woven de baixa porosidade, diferentes técnicas de pré-coagulaçāo e précozimento, próteses impregnadas de colágeno e cerclagem da anastomose distal. Um importante avanço no tratamento da dissecçāo aórtica foi a introduçāo da cola biológica (gelatina-resorcina polimerizada com formaldeído), que representou mudança significativa na prevenção e controle do sangramento per-operatório ${ }^{14}$.

O tubo de pericárdio bovino corrugado é facil de ser manipulado durante a operaçāo e proporciona um conduto circular que mantém a sua forma ci- 
SALLES, C. A.; RIBEIRO, N. A. M.; KALIL, R. A. K.; VIEIRA, G. L.; SOUZA, L. S. S.; BORÉM, P. M.; ANDRADE, M. E. C.; FALEIROS, R. D.; ANDRADE Jr., M. A. M. - Reconstruçāo arterial com tubo de pericárdio bovino corrugado. Rev. Bras. Cir. Cardiovasc., 7(2):88-95, 1992.

líndrica durante o procedimento e facilita a feitura da anastomose. O pericárdio é muito macio e coapta muito bem nas linhas de sutura, resultando uma anastomose hemostática, mesmo em aortas finas e friáveis. Isto é particularmente útil para reimplante das artérias coronárias durante a substituiçāo total da aorta ascendente. Outra vantagem é que esse tubo de pericárdio tem uma superfície não trombogênica, é impermeável, nāo necessita précoagulaçāo e nāo sangra. Sangramento durante a operação com perda sangüínea significativa nāo ocorreu em nenhum paciente desta série; nenhum necessitou reoperaçāo por hemorragia. Quando o tubo corrugado foi utilizado no coraçāo direito, verificou-se que se posiciona e se acomoda muito bem no mediastino. Essas características e vantagens do tubo de pericárdio bovino corrugado foram observadas ao longo da experiência cirúrgica ${ }^{42,44}$.
Esse seguimento não é suficiente para avaliar os resultados a longo prazo, tais como durabilidade, degeneração fibrocálcica, retração e dilatação aneurismática. Entretando, a experiência clínica usando retalho de pericárdio para alargamento da aorta ascendente e tubos de pericárdio nāo corrugado park substituiçāo total da aorta ascendente tem demonstrado bons resultados tardios em seguimentos acima de dez anos ${ }^{4,10}$. A reconstruçăo da via de saída do ventrículo direito com tubos de pericárdio bovino nāo corrugado também tem mostrado bons resultados clínicos durante um seguimento de seis anos ${ }^{36}$. Acreditamos que resultados similares podem ser antecipados com a utilização de condutos de pericárdio bovino corrugado, uma vez que esse processo, como demonstrado por estudos in vitro ${ }^{43}$, năo afeta as características estruturais e funcionais do pericárdio.

SALLES, C. A.; RIBEIRO, N. A. M.; KALIL, R. A. K.; VIEIRA, G. L.; SOUZA, L. S. S.; BORÉM, P. M.; ANDRADE, M. E. C.; FALEIROS, R. D.; ANDRADE Jr., M. A. M. - Arterial reconstruction with crimped bovine pericardial conduit. Rev. Bras. Cir. Cardiovasc., 7(2):88-95, 1992.

ABSTRACT: From August 1989 to February 1992, 32 patients underwent vascular reconstructions using crimped bovine pericardial conduits processed in glutaraldehyde. The introduction of the crimping process used for synthetic vascular prosthesis provided circular tubes, which retain their shape with bending and avoid kinking. Twenty-nine patients (Group I) presented vascular lesions involving the thoracic and/or abdominal aorta, including aneurysms, acute dissections, coarctation and aorto-iliac lesion. The thoracic aortic reconstruction was performed in 25 patients, including aortic valve replacement in 10 , the abdominal aorta in 2 , and the aortic bifurcation in 2 . Three patients (Group II) with complex congenital heart lesions underwent reconstruction of the right ventricular outflow tract in 2, and the Fontan operation in 1 . Hospital mortality in Group I was $24 \%$ (7 patients). Causes of death were low cardiac output in 4, recurrence of aortic dissection in 2 and respiratory infection in 1 . Six of them had been operated upon as emergencies due to acute aortic dissection. All patients in group II survived the operation. There was 1 late death in group I due to methabolic complications related to diabetes and chronic renal failure. This initial clinical experience showed a mean follow-up of 16 months per patient and the longest follow-up was 32 months. It was not observed any late complication related to the crimped bovine pericardial conduit upto-date.

DESCRIPTORS: arterial prosthesis; vascular prosthesis; aneurysms, aortic, surgery.

\section{REFERÊNCIAS BIBLIOGRÁFICAS}

1 ABLAZA, S. G. G.; GHOSH, S. C.; GRANA, V. P. - Use of a ringed intraluminal graft in the surgical treatment of dissecting aneurysms of the thoracic aorta. J. Thorac. Cardiovasc. Surg., 76: 390-396, 1978.

2 AGARWAL, K. C.; EDWARDS, W. D.; FELDT, R. H.; DANIELSON, G. K.; PUGA, F. J.; McGOON, D. C. - Clinicopathological correlates of obstructed rightsided porcine-valved extracardiac conduits. J.
Thorac. Cardiovasc. Surg., 81: 591-601, 1981.

3 ANGELINI, G. D.; WITSENBURG, M.; TEN KATE, F. J. W.; HIDDEMA, P. A. E.; QUAEGEBEUR, J. M. Severe stenotic scar contracture of the Microvel Hemashield right-sided extracardiac conduit. Ann. Thorac. Surg., 48: 714-716, 1989.

4 ARDITO, R. V.; SANTOS, J. L. V.; MAYORQUIM, R. C.; GRECO, O. T.; ZAIANTCHIC, M.; SOTO, H. G.; JACOB, J. L. B.; BRAILE, D. M. - Substituiçāo com- 
SALLES, C. A.; RIBEIRO, N. A. M.; KALIL, R. A. K.; VIEIRA, G. L.; SOUZA, L. S. S.; BORÉM, P. M.; ANDRADE, M. E. C.; FALEIROS, R. D.; ANDRADE Jr., M. A. M. - Reconstruçảo arterial com tubo de pericárdio bovino corrugado. Rev. Bras. Cir. Cardiovasc., 7(2):88-95, 1992.

pleta da aorta ascendente e da valva aórtica com tubo valvulado de pericárdio bovino. Rev. Bras. Cir. Cardiovasc., 2: 129-138, 1987.

5 BENTALL, H. \& DE BONO, A. - Technique for complete replacement of the ascending aorta. Thorax, 23: 338-339, 1968.

6 BLAKEMORE, A. H. \& VOORHEES, A. B. - The use of tubes constructed from Vinyon " $\mathrm{N}$ " cloth in bridging arterial defects: experimental and clinical. Ann. Surg., 140: 324-334, 1954.

7 BORST, H. G. \& SCHAPS, D. - Treatment of extensive aortic aneurysms by a new multiple stage approach. J. Thorac. Cardiovasc. Surg., 95: 11-13, 1988.

8 BORST, H. G.; WALTERBUSCH, G., SCHAPS, D. Extensive aortic replacement using "elephant trunk" prosthesis. J. Thorac. Cardiovasc. Surg., 31: 37 40, 1983.

9 BOWMAN Jr., F. O.; HANCOCK, W. D.; MALM, J. R. - A valve containing Dacron prosthesis: Its use in restoring pulmonary artery-right ventricular continuity. Arch. Surg., 107: 724-728. 1973.

10 BRAILE, D. M.; ARDITO, R. V.; GRECO, O. T. - Alargamento da raíz da aorta com "patch" de pericárdio bovino preservado pelo glutaraldeído. Arq. Bras. Cardiol., 41: 289-296, 1983.

11 BROWN, J. W.; GIROD, D. A.; HURWITZ, R. A.; CALDWELL, R. A.; ROCCHINI, A. P.; BEHRENDT, D. M.; KIRSH, M. M. - Apicoaortic valved conduits for complex left ventricular outflow obstruction: technical considerations and current status. Ann. Thorac. Surg. 38: 162-168, 1984.

BROWN, J. W.; SALLES, C. A.; KIRSH, M. M. Extraanatomical bypass of the aortic root: an experimental technique. Ann. Thorac. Surg., 24: 433438, 1977.

BULLABOY, C. A. - Bovine pericardium: another cautionary note. Ann. Thorac. Surg., 48: 743, 1989.

CABROL, C.; PAVIE, A; MESNILDREY, P.; GANDJBAKHCH, I.; LAUHGLIN, L.; BORS.; CORCOS, T. - Long-term results with total replacement of the ascending aorta and reimplantation of the coronary arteries. J. Thorac. Cardiovasc. Surg., 91: $17-25,1986$.

CASAGRANDE, I.S.J.; PUIG, L. B; SALLES, C. A.; RICARDO, O. P. - Tubo de pericárdio bovino corrugado recoberto com Dacron: novo modelo de prótese vascular (resumo). Rev. Col. Bras. Cir. (Supl.), 15: 153, 1988.

CIARAVELLA Jr., J. M.; D. C.; DANIELSON, G. K.; WALLACE, R. B.; MAIR, D. D.; ILSTRUP, D. M. Experience with the extracardiac conduit. J. Thorac. Cardiovasc. Surg., 78: 920-930, 1979.

pericardium: a source of pulmonary venous obstruction in the Mustard procedure. Ann. Thorac. Surg., 44: 552-553, 1987.

18 CRAWEORD E. S. \& CRAWFORD, J. L. - Diseases of the aorta including an atlas of angiographic pathology and surgical technique. Williams \& Wilkins, Baltimore, 1984

CRAWFORD, E. S.; SVENSON, L. G.; COSSELI, L. S.; SAFI, H. J.; HESS, K. R. - Surgical treatment of aneurysm and/or dissection of the ascending aorta, transverse aortic arch, and ascending aorta and transverse aortic arch. J. Thorac. Cardiovasc. Surg., 98: 659-674, 1989.

CRAWFORD Jr., F. A.; SADE, R. M.; SPINALE, F. Bovine pericardium for correction of congenital heart defects. Ann. Thorac. Surg., 41: 602-605, 1986.

DUBOST, C.; ALLARY, M.; OECONOMOS, N. Ressection of an aneurysm of the abdominal aorta: reestablishment of the continuity by a preserved human arterial graft with result after five months. Arch. Surg., 64: 405-408, 1952.

FONTAN, F.; CHOUSSAT, A.; DEVILLE, C.; DOUTREMEPUICH, C.; COUPILLAND, J.; VOSA, C. - Aortic valve homografts in the surgical treatment of complex cardiac malformations. J. Thorac. Cardiovasc. Surg., 87: 649-657, 1984.

GAGO, O.; SALLES, C. A.; STERN, A. M.; SPOONER, E.; BRANDT, R. L.; MORRIS, J. D.; - A different approach for the total correction of tricuspid atresia. J. Thorac. Cardiovasc. Surg., 72: 209-214, 1976.

GROSS, R. E.; BILL Jr., A. H.; PEIRCE, E. C. - Methods for preservation and transplantation of arterial grafts: observations on arterial grafts in dogs. Report of transplantation of preserved arterial grafts in nine human cases. Surg. Gynecol. Obstet., 88: 689701, 1949.

HOOTS, A. V. \& WATSON Jr., D. C. - Construction of an aortic homograft conduit for right ventricle to pulmonary artery continuity. Ann. Thorac. Surg., 48: $731-732,1989$.

KAY, P. H. \& ROSS, D.N. - Fifteen years's experience with the aortic homograft: the conduit of choice for right ventricular outflow tract reconstruction. Ann. Thorac. Surg., 40: 360-364, 1985.

KIRKLIN, J. W.; BLACKSTONE, E. H.; MAEHARA, T.; PACIFICO, A. D.; KIRKLIN, J. R.; POLLOCK. S.; STEWART, R. W. - Intermediate-term fate of cryopreserved allograft and xenograft valved conduits. Ann. Thorac. Surg., 44: 598-606, 1987. replacement at 20 years: wrap or no wrap? shunt or no shunt? Ann. Thorac. Surg., 48: 615-616, 1989. 
SALLES, C. A.; RIBEIRO, N. A. M.; KALIL, R. A. K.; VIEIRA, G. L.; SOUZA, L. S. S.; BORÉM, P. M.; ANDRADE, M. E. C.; FALEIROS, R. D.; ANDRADE Jr., M. A. M. - Reconstruçāo arterial com tubo de pericárdio bovino corrugado. Rev. Bras. Cir. Cardiovasc., $7(2): 88-95,1992$.

KOUCHOUKOS, N. T.; MARSHALL, W. G.; WEDIGESTECHER, T. A. - Eleven-year experience with composite graft replacement of the ascending aorta and aortic valves. J. Thorac. Cardiovasc. Surg., 92: 691-705, 1986.

LINDENAUER, S. M. - The fabric vascular prosthesis. In: RUTHERFORD, R. B. (ed.) Vascular Surgery, 3. ed. Philadelphia, W. B. Sounders, 1989. Vol. 1, pp 450-460.

LINDENAUER, S. M.; WEBER, T. R.; MILLER, T. A.; RAMSBURG, S. R.; SALLES, C. A.; KAHN, S. P.; WOJTALIK, R. S. - The use of velour as a vascular prosthesis. Biomed. Eng., 11: 301-306, 1976.

McGOON, D. C.; WALLACE, R. B.; DANIELSON, G. K. - Homografts in reconstruction of congenital cardiac anomalies. Mayo Clin. Proc., 47: 101-104, 1972.

MILLER, D. C.; STINSON, E. B.; OYER, P. E.; MORENO-CABRAL, R. J.; REITZ, B. A.; ROSSITER, S. J.; SHUMWAY, N. E. - Concomitant resection of ascending aortic aneurysm and replacement of the aortic valve. J. Thorac. Cardiovasc. Surg., 79: 388-401, 1980.

MOODIE, D. S.; MAIR, D. D.; FULTRON, R. E.; WALLACE, R. B.; DANIELSON, G. K.; McGOON, D. C. - Aortic homograft obstruction. J. Thorac. Cardiovasc. Surg., 72: 553-561, 1976.

MORAES, C. R.; RODRIGUES. J.V.; GOMES, C. A.; MARINUCCI, L.; SANTOS, C. L.; COELHO, T. C.; CAVALCANTI, L. L. - Operaçāo de Rastelli utilizando-se conduto valvulado de pericárdio bovino: experiência inicial. Rev. Bras. Cir. Cardiovasc., 3: 8492, 1988.

NORWOOD, W. L.; FREED, M. D.; ROCCHINI, A. P.; BERNHARD, W. F.; CASTANEDA, A. R. - Experience with valved conduits for repair of congenital cardiac lesions. Ann. Thorac. Surg., 24: 223-232, 1977.

OUDOT, J. - La greffe vasculaire dans les thromboses du carrefour aortic. Presse Med., 59: 234-236, 1951.

PALMA, H.; JULIANO, J. A.; CAL, R. G. R.; ALMEIDA, D. R.; OTA, L. H.; GIANOTTI, I. A.; ANDRADE, J. C. S.; BUFFOLO, E. - Tratamento dos aneurismas

da aorta descendente por endoprótese (tromba de elefante). Rev. Bras. Cir. Cardiovasc., 4: 190-194, 1989.

RASTELLI, G. C.; ONGLEY, P. A.; DAVIS, G.D; KIRKLIN, J. W. - Surgical repair for pulmonary valve atresia with coronary pulmonary artery fistula: report of case. Mayo Clin. Proc., 40: 521-527, 1965.

ROSS, D. N. \& SOMERVILLE, J. - Correction of pulmonary atresia with a homograft aortic valve. Lancet, 2: 1446-1447, 1966.

SALLES, C. A. - Crimped bovine pericardial conduit. Annals of the 2nd World Conference on Open Heart Surgery, Bombay, India, 1991.

SALLES, C. A.; PUIG, L. B.; CASAGRANDE, I. S.; KALIL, R. A.; SOUZA, L. S. S.; ANDRADE Jr.; M. A. M.; - Early experience with crimped bovine pericardial conduit for arterial reconstruction. Eur. J. Cardio-Thorac. Surg., 5: 273-279, 1991.

SALLES, C. A.; PUIG, L. B.; CASAGRANDE, I. S.; VIEIRA, G. L.; KALIL, R. A. - Crimped bovine pericardial conduit for arterial reconstruction. Annals of the 4th Annual Meeting of the European Association for Cardio-Thorac Surgery, Naples, Italy, 1990.

SARAVALLI, O. A.; SOMERVILLE, J.; JEFFERSON, K. E. - Calcification of aortic homografts used for reconstruction of the right ventricular outflow tract. J. Thorac. Cardiovasc. Surg., 80: 909-920, 1981.

SCHLICHTER, A.J. \& KREUTZER, G. O. - Autologous pericardial valved conduit. Rev. Lat. Cardiol. Cir. Cardiovasc. Infan., 1: 43-48, 1985.

SCOTT, S. M.; GODDY, L. R.; SAHMEL, R.; HOFFMAN, H. - A collagen coated vascular prosthesis. J. Cardiovasc. Surg., 28: 498-504, 1987.

STARK, J. - Do we really correct congenital heart defects? J. Thorac. Cardiovasc. Surg., 97: 1-14, 1989.

49 VOORHEES Jr, A. B.; JARETZKI, A.; BLAKEMORE, A. $\mathrm{H}$. - The use of tubes constructed from Vinyon " $\mathrm{N}$ " cloth in bridging arterial defects. Ann. Surg., 135: 332-336, 1952. 